\title{
BDNF-Induced Increase of PSD-95 in Dendritic Spines Requires Dynamic Microtubule Invasions
}

\author{
Xindao Hu, ${ }^{1}$ Lauren Ballo, ${ }^{2}$ Lauren Pietila, ${ }^{2}$ Chris Viesselmann, ${ }^{2}$ Jason Ballweg, ${ }^{2}$ Derek Lumbard, ${ }^{2}$ Matt Stevenson, ${ }^{2}$ \\ Elliott Merriam, ${ }^{1}$ and Erik W. Dent ${ }^{1,2}$ \\ ${ }^{1}$ Neuroscience Training Program and ${ }^{2}$ Department of Neuroscience, University of Wisconsin, Madison, Wisconsin 53706
}

Microtubules (MTs) are capable of entering dendritic spines in mature hippocampal neurons through dynamic polymerization. Although these MT invasions are directly associated with neuronal activity, their function remains unknown. Here we demonstrate in mouse hippocampal neurons that MT entries into spines regulate the increase in postsynaptic density-95 (PSD-95) protein after brain-derived neurotrophic factor (BDNF) treatment. Using multiwavelength total internal reflectance fluorescence microscopy, we show that BDNF prolonged the average MT dwell time in spines and that this effect was dependent on TrkB receptor activation. Further examination revealed that peaks of MT polymerization into spines corresponded to rapid PSD-95 increases in the spine head. Over time, spines targeted by MTs after BDNF application, but not before, showed a robust increase in PSD-95. Conversely, spines completely devoid of MT invasions showed no significant change in the level of PSD-95. Pharmacological inhibition of MT dynamics abolished the BDNF-induced increase in PSD-95. Together, these results support the hypothesis that the well known increase in PSD-95 within spines after BDNF treatment is dependent on MT invasions of dendritic spines. Thus, our study provides a direct link between dynamic MTs and the postsynaptic structure, and provides a functional role for MT invasion of dendritic spines.

\section{Introduction}

In mature cortical and hippocampal neurons, microtubules (MTs) make rapid, infrequent excursions into dendritic spines through dynamic plus end polymerization and depolymerization (Gu et al., 2008; Hu et al., 2008; Jaworski et al., 2009). Furthermore, $\mathrm{MT}$ entries are promoted by neuronal depolarization $(\mathrm{Hu}$ et al., 2008) and pharmacological inhibition of MT dynamics blocks long-term BDNF-induced spine formation ( $\mathrm{Gu}$ et al., 2008). These results indicate that MT entries into dendritic spines may play an important role during brain-derived neurotrophic factor (BDNF)-induced synaptic plasticity. However, the mechanism that links BDNF-induced spine plasticity and MT invasions remains unknown.

The postsynaptic density-95 (PSD-95) scaffolding protein has been identified as a marker for synaptic strength. Overexpression of PSD-95 promotes synaptic maturation (El-Husseini et al., 2000), while knockdown of PSD-95 results in decreased synaptic strength and spine density (Ehrlich et al., 2007). PSD-95 remains

Received May 5, 2011; revised Sept. 9, 2011; accepted Sept. 15, 2011.

Author contributions: X.H. and E.W.D. designed research; X.H., L.B., L.P., C.V., J.B., D.L., and M.S. performed research; E.M. and E.W.D. contributed unpublished reagents/analytic tools; X.H., L.B., and L.P. analyzed data; X.H. and E.W.D. wrote the paper.

This work was supported by grants from NIH (R01-NS064014), Dana Foundation, and Whitehall Foundation to E.W.D. We thank Katherine Kalil, Tim Gomez, and all of the members of the Dent laboratory for helpful discussions and comments on the manuscript. We also thank Katherine Kalil for the use of the Amaxa electroporator. mCherry$\alpha$-tubulin in a 11 vector (Clontech) was kindly provided by Stephanie Gupton. GFP-PSD-95 was a generous gift from David Bredt.

Correspondence should be addressed to Erik W. Dent, Department of Neuroscience, University of Wisconsin, 1300 University Avenue, 332 SMI, Madison, WI 53706.E-mail: ewdent@wisc.edu.

DOI:10.1523/JNEUROSCI.2445-11.2011

Copyright $\odot 2011$ the authors $\quad 0270-6474 / 11 / 3115597-07 \$ 15.00 / 0$ mobile in mature neurons, and can transit in and out of spines on the order of minutes (Marrs et al., 2001). A pool of dendritic PSD-95 molecules is shared and redistributed among neighboring spines through diffusion (Gray et al., 2006; Tsuriel et al., 2006). In visual cortical neurons, BDNF can increase the size of PSD-95 puncta in spines and the overall amount of PSD-95 in dendrites within $60 \mathrm{~min}$ (Yoshii and Constantine-Paton, 2007). Thus, we hypothesized that BDNF increases the levels of PSD-95 in spines through the regulation of dynamic MT invasions, and these invasions directly contribute to the accumulation of PSD-95.

To test this possibility, we used total internal reflection fluorescence microscopy (TIRFM) to simultaneously monitor MT dynamics and PSD-95 levels in dendritic spines of mature hippocampal neurons. TIRFM allowed us to obtain high-signal-tonoise fluorescence images at rapid intervals for long periods of time (Hu et al., 2008). TIRFM has also been shown to effectively capture changes in PSD-95 clusters by eliminating background signals from cytosolic PSD-95 molecules (Noritake et al., 2009). Here, we discovered that BDNF treatment increased the MT dwell time in dendritic spines. MT polymerization into spines also correlated with rapid increases of PSD-95 in the spine head. Within 20 min after BDNF treatment, only spines invaded by MTs showed a significant increase of PSD-95 levels, which was positively correlated with the probability that an MT was present in that spine. Moreover, pharmacological inhibition of MT dynamics effectively blocked the BDNF-induced increase in PSD95. Together, our results indicate that MTs directly contribute to postsynaptic structural changes by regulating the increase of PSD-95 in spines after BDNF treatment. 


\section{Materials and Methods}

Plasmids and reagents. DsRed 2 and EGFP- $\alpha$ tubulin in $\mathrm{PCAX}$ vectors were described previously (Hu et al., 2008). Newly polymerized microtubules were stained with rat antityrosinated tubulin at 1:1000 (YL1/2 clone, Millipore). Alexa-conjugated phalloidin (1:50) and Alexa-conjugated anti-rat secondary antibody (1:500) were from Invitrogen. BDNF was from Promega; and K252a, nocodazole, and taxol were from Sigma-Aldrich.

Cell culture and transfection. All mouse procedures were approved by the University of Wisconsin Committee on Animal Care and were in accordance with NIH guidelines. E15.5 hippocampal neuron cultures were prepared from Swiss Webster mice of either sex (Taconic) and transfected as described previously (Hu et al., 2008), with modifications (Viesselmann et al., 2011).

Live-cell TIRF imaging and immunocytochemistry. All imaging was performed at physiological temperatures $\left(36-37^{\circ} \mathrm{C}\right)$ on a Nikon TE2000E microscope as described previously (Hu et al., 2008). Neurons were fixed and stained as described previously (Dent et al., 2007). Images of fixed neurons were collected with TIRFM at a resolution of $0.063 \mu \mathrm{m} /$ pixel. All experiments were repeated with at least three individual primary cell cultures.

Quantification of microtubule dynamics and levels of PSD-95. Dual-wavelength time-lapse images were acquired at $10 \mathrm{~s}$ intervals for 20 min before and after the addition of BDNF with and without MT drugs, generating image stacks of 241 frames. A maximum projection image of the tubulin channel was generated by overlaying a stack into one image (121 frames, before or after treatment). An average projection image of PSD-95 was created to depict the morphology of dendritic protrusions. Live-cell images were collected at a resolution of 0.126 $\mu \mathrm{m} /$ pixel. For clarity, PSD-95 is shown in red and tubulin is shown in green in all figures. MT invasions into spines were determined by examining the time-lapse movies frame by frame. Parameters of MT invasions into dendritic spines were measured by tracking the polymerizing/depolymerizing plus end of MTs in image stacks with MetaMorph software (Molecular Devices) and calculated in Excel (Microsoft). MT invasion tracings were created by plotting the MT polymerization distance into spines (distance to origin) over time.

Kymographs of PSD-95 were generated by measuring the average fluorescence intensity (AFI) within the width of a 4-pixel-wide line drawn across the long axis of the puncta. To measure PSD-95 levels, a region of interest was drawn around the entire PSD-95 puncta in the frame where it attained its largest area. The AFI of PSD-95 within this region was measured at each time point. In Figure 3E, spine volume was calculated by averaging the AFI of DsRed over 1 min ( 6 frames) at each time point. For the analysis in Figure 3, F-I, and Figure 4, PSD-95 level was calculated by measuring the AFI over 1 min. Only spines with a distinct PSD-95 punctum in the spine head were analyzed. Control spines included in Figure $3 F$ were chosen from the same dendritic segment as neighboring MT-targeted spines. The percentage chance of an MT being present in the spine (see Fig. $3 J$ ) was determined by calculating the probabilities of finding an MT in all MT-targeted spines at each individual time point (241 time points in each 40 min time lapse). Spines in different categories (see Fig. $3 F-I$ ) generated different values of the "percentage chance of phalloidin

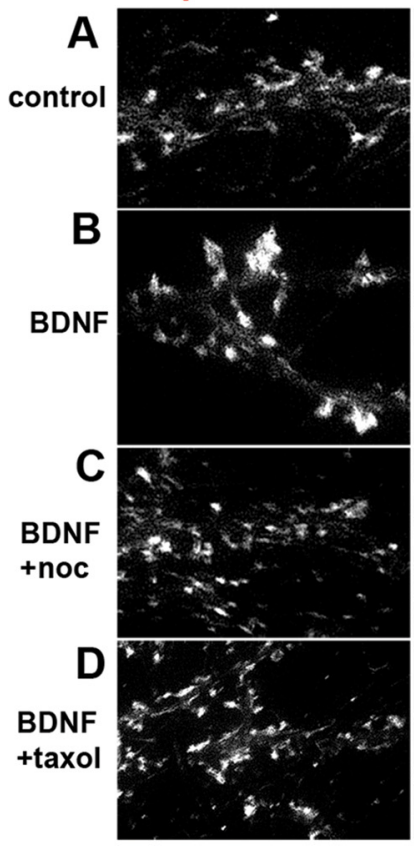

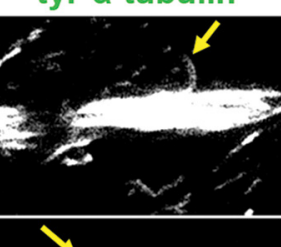
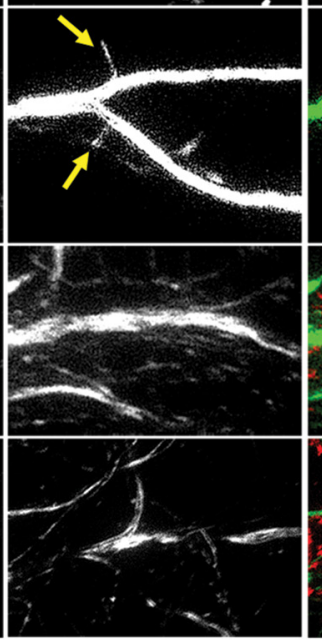

.

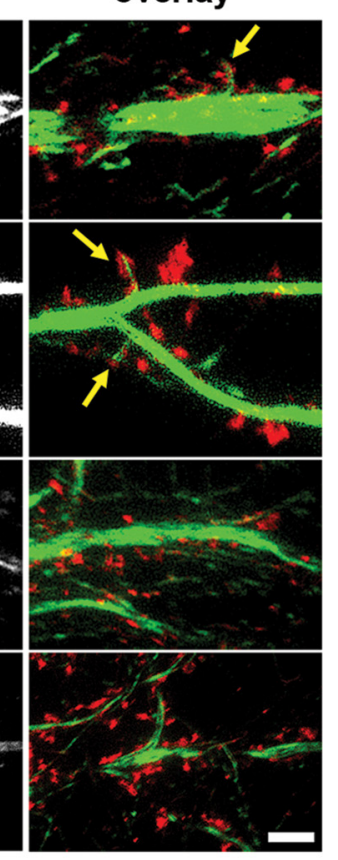

Figure 1. BDNF treatment increases endogenous MT entry into spines. $\boldsymbol{A}$, Tyrosinated $\alpha$-tubulin antibody-labeled MTs (yellow arrows) are detected in a small percentage of F-actin-labeled spines under basal conditions (21-28 DIV). $\boldsymbol{B}$, After a 20 min of 100 nm nocodazole $(\boldsymbol{C})$ or $10 \mathrm{~nm}$ taxol $(\boldsymbol{D})$ to the BDNF solution inhibits MT invasions. $\boldsymbol{E}$, Quantification of the treatments (Kruskal-Wallis test with Dunn's post hoc tests—-see Materials and Methods for significance values). Scale bar: (in D) A-D, $5 \mu \mathrm{m}$.

MT in spine." Time points with the same percentage chance of MT in spine value were grouped together. The percentage change in PSD-95 of all spines at these time points were then compiled into each data point.

Statistics and graphing. All statistical tests were performed with GraphPad Prism. For each dataset, parametric or nonparametric statistical tests and post hoc tests were performed depending on the variance of the datasets. In all graphs $p$ values are depicted as follows: ${ }^{\star} p<0.05,{ }^{\star *} p<$ $0.01,{ }^{* *} p<0.001$. Graphs were compiled in Prism.

\section{Results}

\section{BDNF prolongs MT invasions into dendritic spines}

Because BDNF increases PSD-95 in dendritic spines of visual cortical neurons (Yoshii and Constantine-Paton, 2007), we hypothesized that dynamic MT invasions into hippocampal dendritic spines could regulate the BDNF-induced increase of PSD95. We first examined the effect of BDNF on the presence of endogenous MTs in dendritic spines by treating 3-week-old hippocampal cultures with either $50 \mathrm{ng} / \mathrm{ml}$ BDNF alone or BDNF with low concentrations of MT drugs for $20 \mathrm{~min}$. We used either 

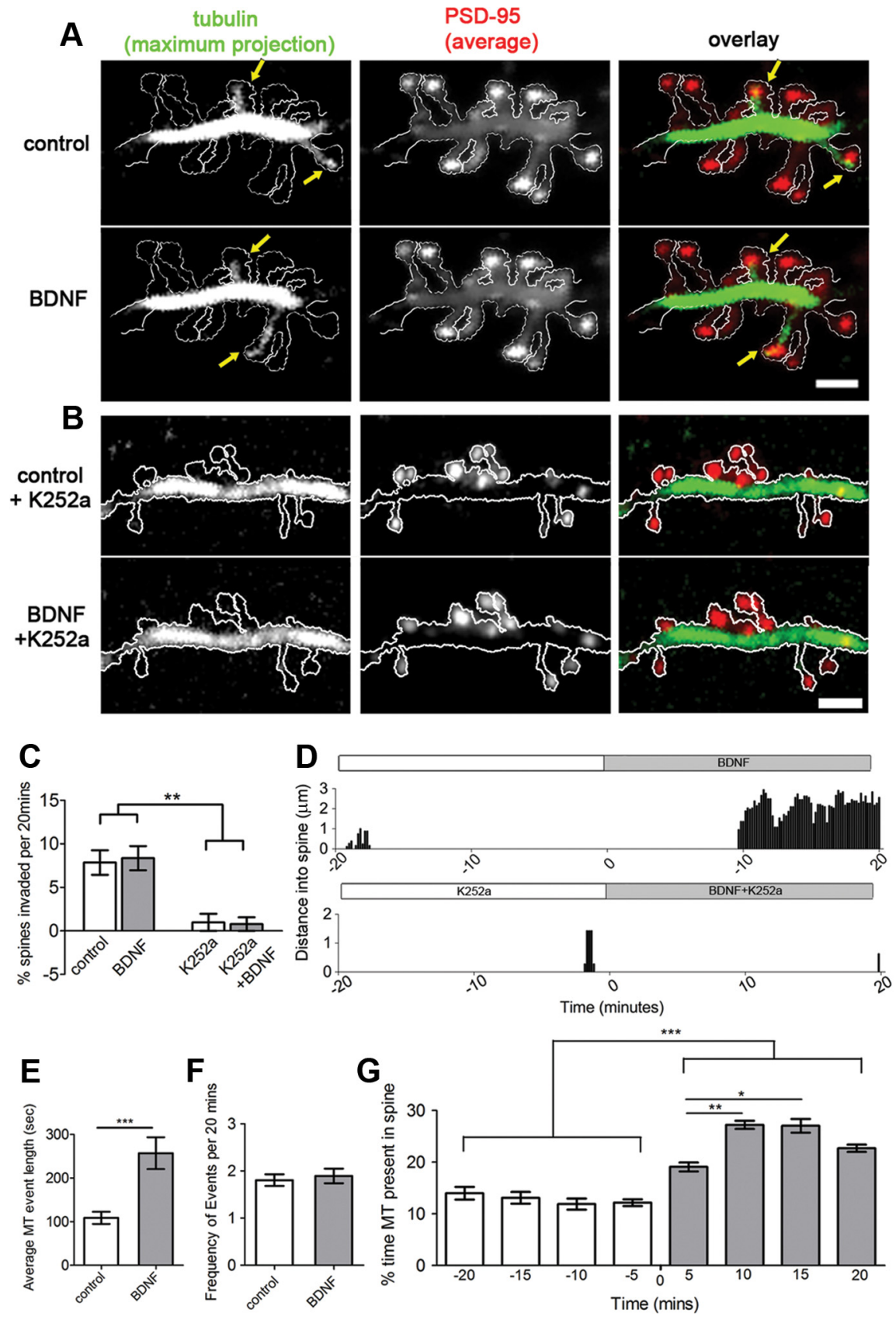

Figure 2. Live-cell TIRFM shows increased MT invasions into spines treated with BDNF. $A, A$ segment of dendrite from a 16 DIV hippocampal neuron, transfected with mCherry- $\alpha$-tubulin and GFP-PSD-95, was imaged for 20 min before (control) and 20 min after addition of BDNF. Maximum projection of tubulin fluorescence depicts spines that were invaded by MTs (yellow arrows) during the time lapse. Average projection of PSD-95 fluorescence acts as a volume fill. B, A dendritic segment of a different neuron incubated with the tyrosine kinase inhibitor K252a for $24 \mathrm{~h}$ before the experiment. C, BDNF treatment does not change the percentage of spines invaded by MTs, whereas K252a decreases MT invasions (two-way ANOVA test with Bonfferoni post-tests). $\boldsymbol{D}$, Examples of MT invasions into two dendritic spines. $\boldsymbol{E}, \boldsymbol{F}$, Average MT invasion event length is significantly increased after BDNF treatment $(\boldsymbol{E})$, while the frequency of invasions among MT-targeted spines remains the same $(\boldsymbol{F})$ (Student's $t$ test). $\boldsymbol{G}$, MT dwell time is elevated within 5 min of BDNF treatment and peaks at 10-15 min (Kruskal-Wallis test with Dunn's post hoc tests). Scale bars, $2 \mu \mathrm{m}$.

$10 \mathrm{~nm}$ taxol or $100 \mathrm{~nm}$ nocodazole to specifically inhibit MT dynamic instability, the rapid polymerization and depolymerization of MT plus ends (Dent and Kalil, 2001). After fixation, neurons were stained with an antibody against tyrosinated $\alpha$-tubulin to label newly polymerized MTs, and phalloidin to label F-actinrich dendritic spines (Fig. $1 A-D$ ). MTs were present in $0.8 \pm$ $0.4 \%$ (all data presented are mean \pm SEM) of spines under control condition ( $n=555$ spines, 7 neurons), consistent with our previous study ( $\mathrm{Hu}$ et al., 2008). Surprisingly, after BDNF treatment $3.8 \pm 0.3 \%$ of spines contained MTs ( $n=768$ spines, 9 neurons). The inclusion of either nocodazole or taxol abolished the increase of MTs in spines (Fig. $1 C-E)$, resulting in $1.4 \pm 0.3 \%$ of spines containing MTs $(n=1030$ spines, 9 neurons) for BDNF plus nocodazole and $1.4 \pm 0.4 \%$ for BDNF plus taxol $(n=769$ spines, 8 neurons). These results indicate that BDNF markedly increases MT invasion of dendritic spines within $20 \mathrm{~min}$, and that these invasions are dependent on MT plus end dynamics.

We then investigated the effects of BDNF on MT dynamics in live cells using TIRFM. With TIRFM we can image cultured hippocampal neurons at $10 \mathrm{~s}$ intervals for $>60 \mathrm{~min}$ without inducing photo damage ( $\mathrm{Hu}$ et al., 2008). Importantly, neurons were transfected before plating, which allowed them to mature while expressing both GFP-PSD-95 and mCherry$\alpha$-tubulin. After 2-3 weeks in culture, we selected only neurons that were expressing relatively low levels of fluorescent proteins (the middle $25-75 \%$ brightest neurons) to minimize overexpression artifacts and phototoxicity. In these studies, we imaged labeled neurons for $20 \mathrm{~min}$ before and after the application of BDNF, resulting in a $40 \mathrm{~min}$ time lapse. Treatment with BDNF increased the time MTs remained in individual spines (average MT event length) from $108 \pm 14$ to $257 \pm$ 37 s ( $n=101$ spines, 7 neurons) [Fig. $2 A, D$ (top),E]. However, BDNF application did not increase the percentage of spines invaded by MTs (before, $7.9 \pm$ $1.4 \%$; after, $8.3 \pm 1.5 \% ; n=1317$ spines, 7 neurons) (Fig. $2 A, C$ ) or the frequency of MT invasion (before, $1.9 \pm 0.2$ events $/ 20$ min; after, $1.9 \pm 0.2$ events $/ 20 \mathrm{~min} ; n=$ 101 spines, 7 neurons) (Fig. $2 F$ ). To examine the temporal change in MT dynamics in spines, we measured the percentage of time during each 5 min time period that MTs were present in the MTtargeted spines ( $n=101$ spines) (Fig. $2 G$ ). Within 5 min of BDNF treatment, the percentage of time that MTs were present in spines was significantly elevated compared with 5 min before BDNF application. This percentage peaked at $10-15$ min after BDNF at a level twice that before BDNF application. These data demonstrate that BDNF treatment increases the percentage of time MTs dwell in dendritic spines, resulting in a higher likelihood that MTs would be present in fixed dendritic spines (Fig. $1 E$ ).

To determine whether the BDNF receptor TrkB was responsible for the BDNF-induced increase in MT dwell time, we pre- 

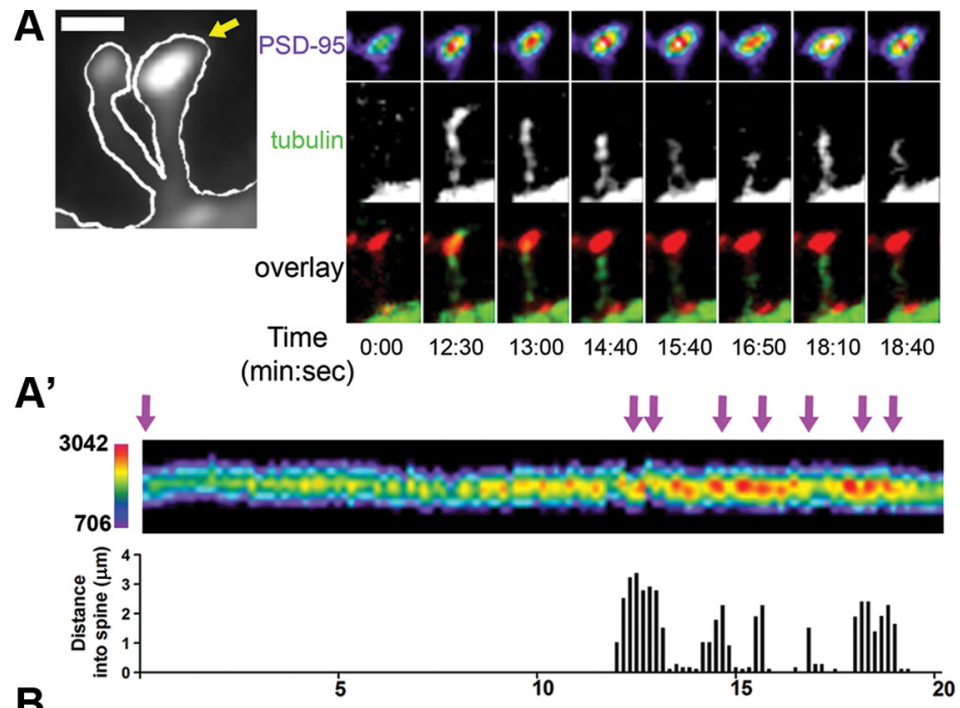

B
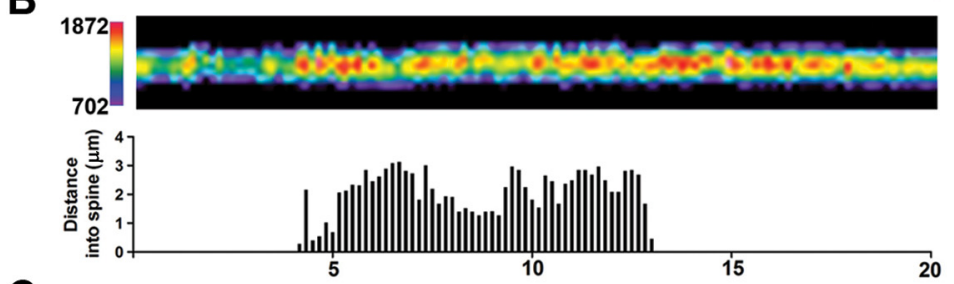

C

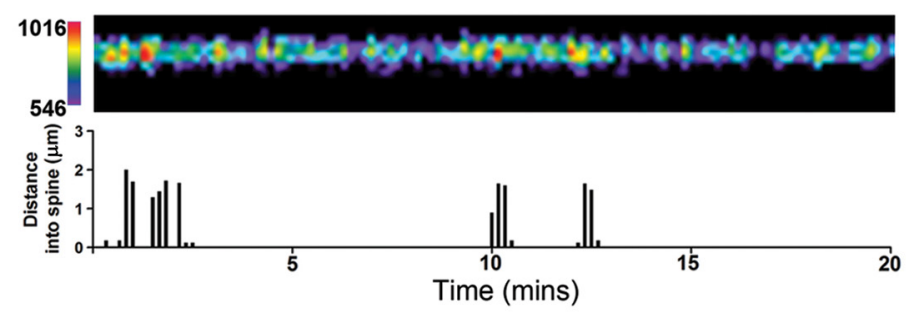

D

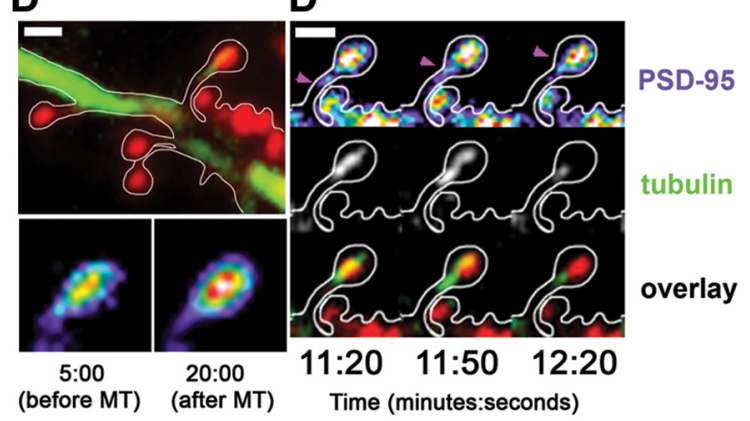

E
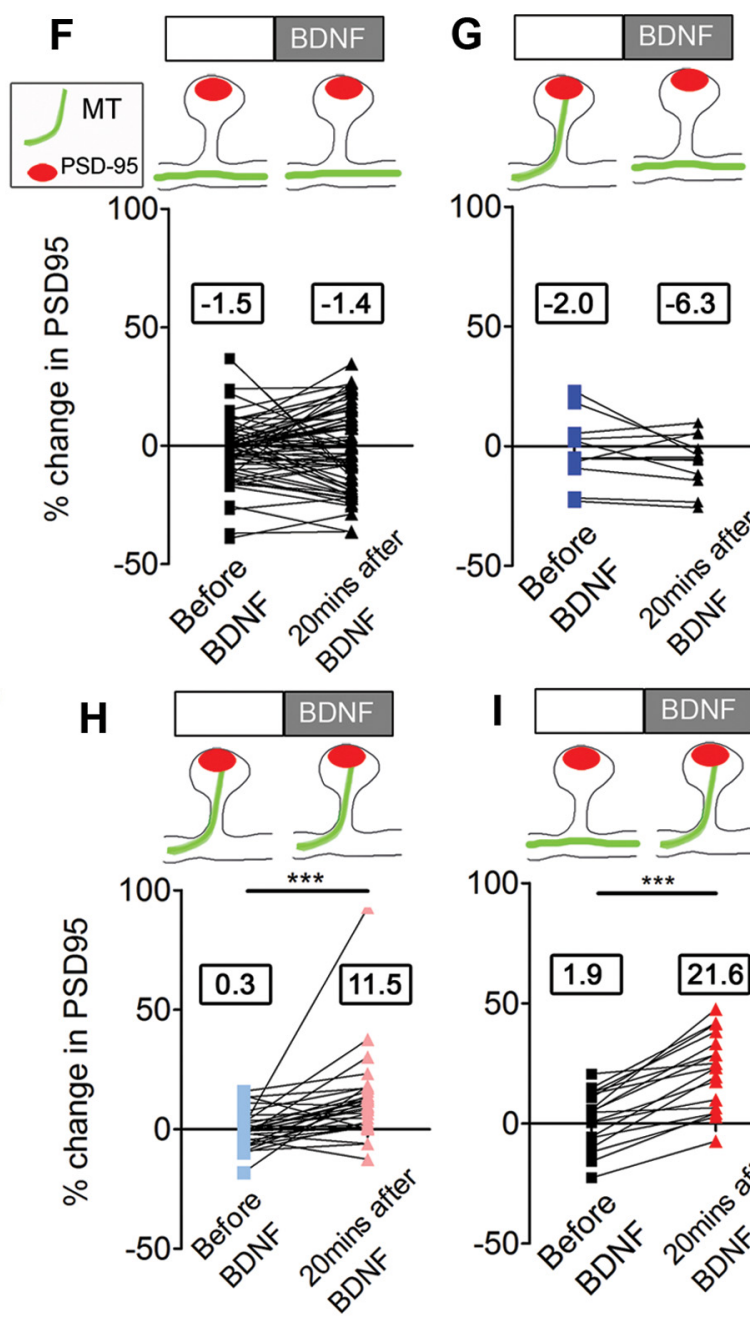

100
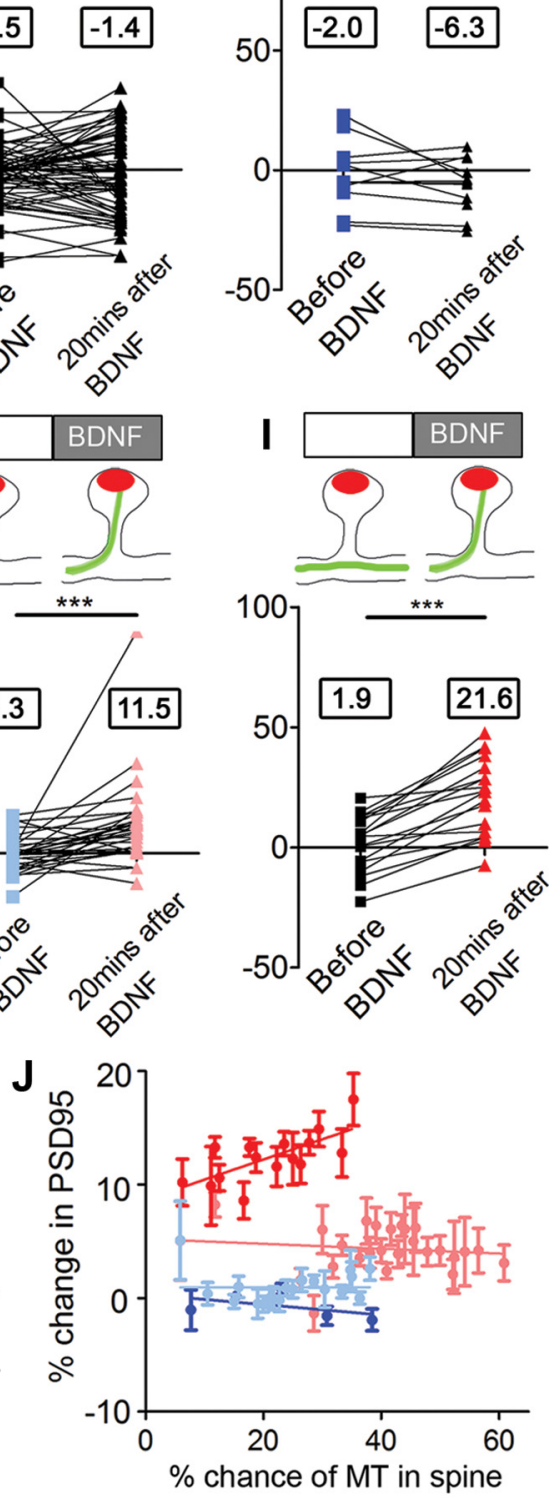

Figure 3. MT invasion of spines is correlated with BDNF-induced increase of PSD-95.A, An example of two hippocampal dendritic spines transfected with GFP-PSD-95 and mCherry- $\alpha$-tubulin. The spine on the right is shown in a series of time-lapse images during MT entries; these time points correspond to purple arrows in $\boldsymbol{A}^{\prime}$. $\boldsymbol{A}^{\prime}, \mathbf{A}$ kymograph of the PSD-95 punctum in the spine shown in $\boldsymbol{A}$. $\boldsymbol{B}, \boldsymbol{C}$, Two other examples of MT-invaded spines after BDNF treatment. $\boldsymbol{D}$, A section of dendrite from a neuron transfected as in $\boldsymbol{A}$. $\boldsymbol{D}^{\prime}$, A series of images showing transport of a PSD-95 punctum (top, purple arrowheads) into a spine head. $E$, Spines show no enlargement 20 min after BDNF treatment. $F, G$, No significant change in PSD-95 level was detected at 20 min after BDNF treatment in spines devoid of MTs or in spines invaded by MTs only during the control time period. $\boldsymbol{H}, \boldsymbol{I}$, PSD-95 levels increased significantly both in spines that had MT entry before and after BDNF $(\boldsymbol{H})$ and only after BDNF (I) (two-tailed Wilcoxon signed rank test). Numbers in black boxes $(\boldsymbol{F}-\boldsymbol{I})$ show the average value of each dataset. Symbol colors in $\boldsymbol{F}-\boldsymbol{H}$ correspond to line colors in $\boldsymbol{J}$. , Percentage change in PSD-95 positively correlates with the percentage chance of an MT being present in a spine only in spines that experience post-BDNF MT invasions. (nonparametric correlation Spearman's $r=0.6845, p<0.01$ ). Scale bars: $A, 2 \mu \mathrm{m} ; \mathbf{D}, \boldsymbol{D}^{\prime}, 1 \mu \mathrm{m}$.

incubated neurons with the tyrosine kinase inhibitor K252a (200 $\mu \mathrm{M})$ for $24 \mathrm{~h}$ to block TrkB activation. Time-lapse imaging revealed that K252a-treated neurons had very few spines invaded by MTs either without $(1.0 \pm 1.0 \%)$ or with $(0.8 \pm 0.8 \%) \mathrm{BDNF}$ treatment ( $n=501$ spines, 4 neurons) (Fig. $2 B, C$ ), both conditions being significantly different from controls (Fig. 2C). In the handful of spines that contained MTs, MT invasions were scarce and transient (Fig. 2D, bottom). Thus, intrinsic signal- ing through the TrkB receptor is essential for basal levels of dynamic MT invasions into dendritic spines.

MT dynamics directly regulate the BDNF-induced increase in PSD-95

To study the possible correlation between MT entries and changes in PSD-95 after BDNF, we monitored MT dynamics and PSD-95 levels simultaneously in individual spines that contained 

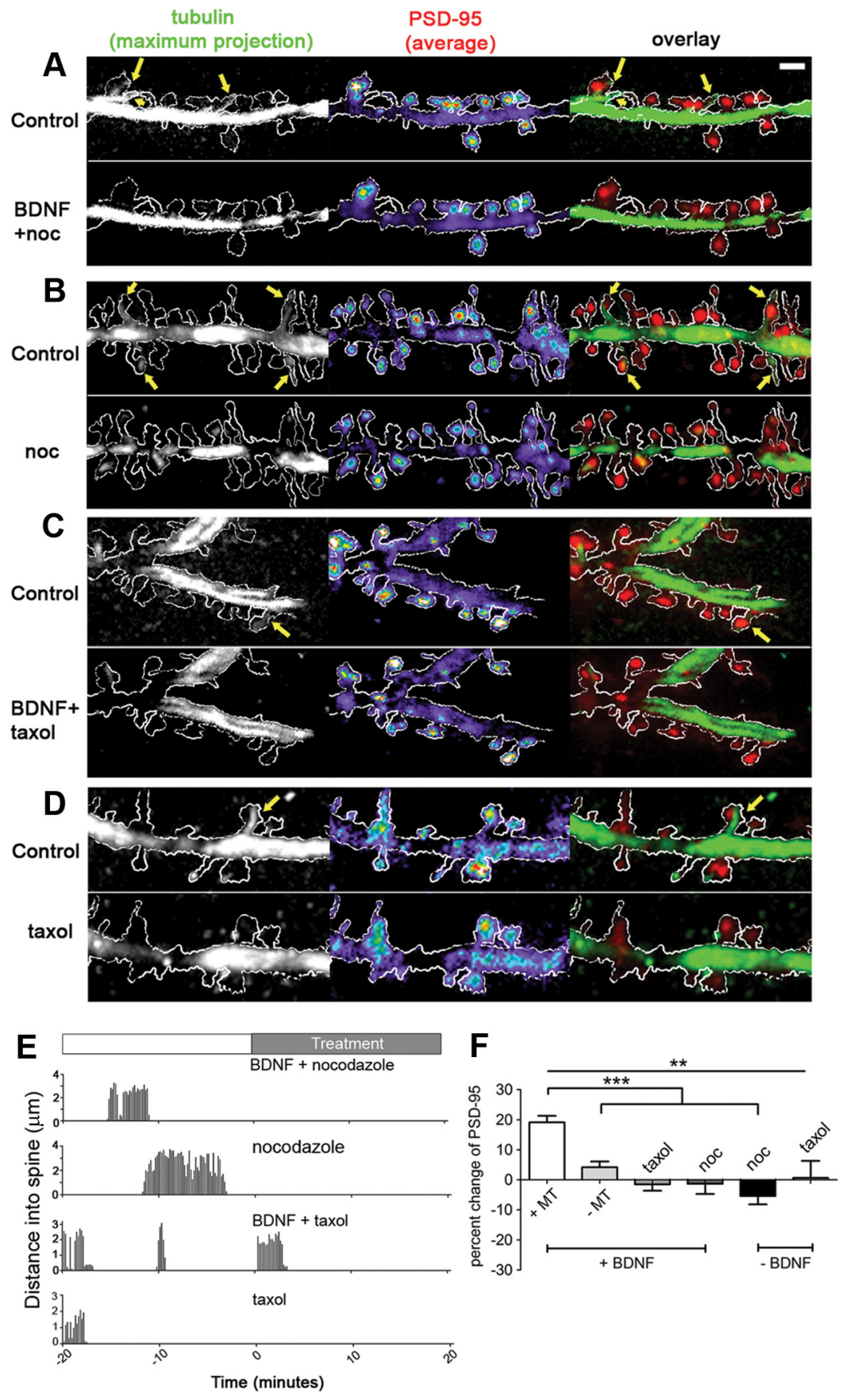

Figure 4. BDNF-induced increase in PSD-95 is abolished by inhibition of MT dynamics. $A-D$, Images of maximum projection of tubulin and average projection of PSD-95 from time-lapse images, with overlays, before and after BDNF and MT drug treatments. By dampening MT dynamics, MT polymerization into spines (yellow arrows) was almost completely blocked. $\boldsymbol{E}$, Examples of traces of MT invasions within individual spines. $\boldsymbol{F}$, Quantification shows no change of PSD-95 level when nocodazole or taxol were included with or without BDNF (Kruskal-Wallis test with Dunn's post hoc tests). Scale bar: (in $\boldsymbol{A}) \boldsymbol{A}-\boldsymbol{D}, 2 \mu \mathrm{m}$.

a distinct PSD-95 punctum in the spine head. MT invasions correlate with "hotspots" of elevated PSD-95 levels (Fig. $3 A, A^{\prime}$ ), which may or may not persist following MT invasion (Fig. $3 A^{\prime}-$ C). Since MTs function as a major route of transport for a variety of intracellular cargoes in all cells, we asked whether PSD-95 is directly transported on MTs during MT entries. We examined 982 individual spines during time-lapse imaging (total imaging time, $4 \mathrm{~h}$ ) and found only one case in which MT invasion accompanied the movement of a PSD-95 punctum into a spine head (Fig. $3 D^{\prime}$ ), followed by a subsequent increase in PSD-95 intensity in that spine head (Fig. 3D). We also documented four cases of a PSD-95 punctum moving into a spine when no MT was present (data not shown). Thus, although MT entries into spines correlated with increases in PSD-95, MTs rarely served as the direct conduit for PSD-95 transport into spines.

To better characterize the correlation between MT polymerization into spines and changes in PSD-95, we measured the average fluorescence intensity of PSD-95 in spine heads by drawing a region of interest (ROI) that included the entire PSD-95 punctum. We found that at the peak of each MT invasion, the PSD-95 level within the ROI increased by $3.0 \pm$ $0.4 \%$ compared with the average of $1 \mathrm{~min}$ before this time point ( $n=206 \mathrm{MT}$ entries; 87 spines, 5 neurons). Conversely, in dendritic protrusions that did not contain a distinct PSD-95 punctum (immature spines), levels of PSD-95 remained unchanged at the peak of MT invasion (data not shown). Thus, MT invasions (red channel) do not cause bleed-through fluorescence into the PSD-95 (green) channel.

To test the hypothesis that MT invasions are responsible for the accumulation of PSD-95, we measured the PSD-95 level in spines over a $1 \mathrm{~min}$ interval at three time points during the $40 \mathrm{~min}$ time lapse; the start of control time period, just before and 20 min after BDNF addition. We measured DsRed2 intensity (as a volume marker) in spines but found no spine enlargement 20 min after BDNF treatment in MT-targeted spines, as well as the general population of spines (Fig. 3E). We then compared the level of PSD-95 (normalized to the start time point) over time in spines with different dynamic MT profiles. Surprisingly, we found no significant change in PSD-95 level in spines devoid of MTs before or after BDNF addition (Fig. $3 F)(-1.5 \pm$ $1.6 \%$ before; $-1.4 \pm 2.0 \%$ after; $n=68$ spines, 7 neurons), as well as spines that were invaded by MTs before but not after BDNF (Fig. $3 G)(-2.0 \pm 3.9 \%$ before; $-6.3 \pm 3.2 \%$ after; $n=12$ spines, 7 neurons). However, the level of PSD-95 increased significantly in spines invaded by MTs both before and after BDNF (Fig. $3 H$ ) $(-0.3 \pm 1.3 \%$ before; $11.5 \pm 3.2 \%$ after; $n=31$ spines, 7 neurons). Interestingly, PSD-95 exhibited the most robust increase in spines invaded by MTs only after BDNF treatment (Fig. $3 I)(1.9 \pm 2.8 \%$ before; $21.6 \pm 3.7 \%$ after; $n=19$ spines, 7 neurons). The increase in PSD-95 in this particular group of spines (Fig. 3I) was significantly higher than in all of the other three groups (Fig. 3G-I) (two-way ANOVA with Bonferroni post-tests, $p<0.01$ ). No significant change in PSD-95 was detected during the control time period among all four groups of spines (two-way ANOVA with Bonferroni post-tests, $p>0.05$ ). Together, these data indicate that MT entries 
that occur after BDNF application correlate with the accumulation of PSD-95. To further test whether the likelihood of MT invasion in a spine scales with the percentage change in PSD-95, we plotted the data for each of the different MT invasion scenarios mentioned above (Fig. 3F-I) and discovered that only when MT entry occurred after BDNF application (Fig. 3J, red line) did the likelihood of an MT in a spine increase linearly with the change in PSD-95. Thus, we conclude that when BDNF causes MTs to polymerize into previously unoccupied spines, the probability of finding MTs in these spines positively correlates with an increase in PSD95, and that over time these spines exhibit the most robust increase in PSD-95 in the spine head.

To determine whether MT dynamics and invasion into spines were necessary for regulating the level of PSD-95, we used nocodazole or taxol to block MT invasions of spines and acquired time-lapse images to monitor the level of PSD-95 and MT dynamics. We discovered that inclusion of either nocodazole (100 $\mathrm{nM})$ or taxol (10 nM) greatly diminished MT invasions after BDNF (Fig. 4A-D); only a few brief entries of MTs occurred in some spines (Fig. 4E). These drug treatments abolished the BDNF-induced increase in PSD-95 (Fig. 4F). The percentage change of PSD-95 after 20 min in BDNF plus taxol or BDNF plus nocodazole was $-1.5 \pm 2.1 \%$ ( $n=31$ spines, 3 neurons $)$ and $-1.3 \pm 3.4 \%$ ( $n=17$ spines, 3 neurons), respectively. Moreover, treatment with taxol or nocodazole alone did not result in a significant change in PSD-95 [0.6 $\pm 5.7 \%(n=13$ spines, 3 neurons) and $-5.4 \pm 2.8 \%$ ( $n=13$ spines, 3 neurons), respectively]. These data show that MT dynamics are necessary for the accumulation of PSD-95 in spines following BDNF treatment and suggest that MT invasion of spines plays an important role in this process. Nevertheless, the exact contributions of dendritic MT dynamics versus MT entries into spines will require the localized disruption of dynamic MTs only in the postsynaptic spine.

\section{Discussion}

In this study, we discovered that BDNF rapidly increased the dwell time of MTs in dendritic spines, that MT invasions were temporally and spatially associated with increases in the level of PSD-95 in mature spines, and that MT dynamics are required for BDNF-induced PSD-95 accumulation in dendritic spines. These results provide the first direct evidence that dynamic MT entries correlate with changes in the postsynaptic molecular composition during BDNF-induced synaptic plasticity. Previous studies have established that MTs remain dynamic in spines throughout the lifetime of neurons (up to 63 DIV) (Hu et al., 2008). Therefore, it is reasonable to speculate that MT invasions of spines play an important role throughout the life of hippocampal neurons by dynamically regulating levels of PSD-95 in spine heads in response to increases in BDNF.

Long-term BDNF stimulation ( $24 \mathrm{~h})$ increases spine density in hippocampal slices and dissociated cultures (Tyler and PozzoMiller, 2001; Gu et al., 2008; Ji et al., 2010), and induces more spines with larger spine heads in mature neurons (Ji et al., 2010). Furthermore, short-term BDNF treatment facilitates LTP induction when paired with electrical stimulation (Kovalchuk et al., 2002; Rex et al., 2007). In this study, we focused on the short-term effects of BDNF treatment. We did not observe significant changes in spine density, shape, or volume within $20 \mathrm{~min}$ of BDNF application. However, MT invasions into spines increased within 5 min of BDNF treatment, indicating that changes in MT dynamics precede longer-term structural changes of these spines (Ji et al., 2010). However, we have not shown here that MT invasions directly precede spine structural changes induced by BDNF in individual spines. The effects of BDNF on LTP maintenance and stabilization of spine growth depend on TrkB activation (Kovalchuk et al., 2002; Rex et al., 2007; Tanaka et al., 2008). We discovered that preincubation of cultures with the tyrosinekinase inhibitor K252a not only eliminated the elevation of MT invasions following BDNF application, but also significantly reduced baseline MT entries into spines. These results suggest that activation of the BDNF-TrkB signaling pathway regulates MT dynamics both under basal conditions and after application of exogenous BDNF.

Apart from its effects on structural spine plasticity, BDNF has also been shown to directly increase the size and average fluorescence intensity of PSD-95 puncta in spines within $60 \mathrm{~min}$ (Yoshii and Constantine-Paton, 2007). Time-lapse imaging studies have revealed that although a subpopulation of PSD-95 puncta are stable in spines for days, individual PSD-95 clusters appear and disappear from spines on the order of minutes to hours (Marrs et al., 2001; Gray et al., 2006; Tsuriel et al., 2006), indicating that these molecules remain dynamic in mature neurons. Using twophoton uncaging in the developing neocortex, median retention time of PSD-95 in spines was discovered to increase from $\sim 22$ to $\sim 100$ min from postnatal day 10 (P10) to P70 in mice (Gray et al., 2006). It was proposed that the mobility of PSD-95 was due to diffusion and primarily regulated by PSD-95-interacting proteins in the spine head, such as CaMKII and Shank. Here we provide a novel mechanism whereby MT invasions regulate PSD-95 in the spine head. We discovered that spines invaded by MTs only after BDNF treatment showed a robust increase in PSD-95 within 20 $\min$. When a polymerizing MT enters a spine during an activated BDNF signaling cascade, it may cause either an increased capture of diffusive PSD-95 or prolonged retention of PSD-95, ultimately leading to accumulation of PSD-95 in the spine head. Nevertheless, how the MT-induced increase in PSD-95 temporally correlates with spine growth remains to be tested.

Another intriguing possibility is that PSD-95 molecules are directly associated with the plus tip protein EB3 during polymerization (Sweet et al., 2011). This could result in a polymerizing MT "delivering" more PSD-95 into spine heads during MT invasions, without PSD-95 appearing as a distinct punctum. If this was the case, PSD-95 should appear concentrated, to some degree, at the tips of polymerizing MTs. We did not observe this in any of our studies and therefore favor a model in which MTs are delivering other, unknown cargo that causes PSD-95 to be captured or retained in the spine head. A recent study has identified a mechanism for PSD-95 movement out of spines via binding to neuroligin 1, which in turn exits spines through dynein-based transport (Schapitz et al., 2010). Although not demonstrated directly, it is likely that this exit of neuroligin/PSD-95 occurs on MTs. However, whether invading MTs traffic cargo directly into spines remains unclear.

\section{References}

Dent EW, Kalil K (2001) Axon branching requires interactions between dynamic microtubules and actin filaments. J Neurosci 21:9757-9769.

Dent EW, Kwiatkowski AV, Mebane LM, Philippar U, Barzik M, Rubinson DA, Gupton S, Van Veen JE, Furman C, Zhang J, Alberts AS, Mori S, Gertler FB (2007) Filopodia are required for cortical neurite initiation. Nat Cell Biol 9:1347-1359.

Ehrlich I, Klein M, Rumpel S, Malinow R (2007) PSD-95 is required for activity-driven synapse stabilization. Proc Natl Acad Sci USA 104:4176-4181.

El-Husseini AE, Schnell E, Chetkovich DM, Nicoll RA, Bredt DS (2000) PSD-95 involvement in maturation of excitatory synapses. Science 290:1364-1368. 
Gray NW, Weimer RM, Bureau I, Svoboda K (2006) Rapid redistribution of synaptic PSD-95 in the neocortex in vivo. PLoS Biol 4:e370.

$\mathrm{Gu}$ J, Firestein BL, Zheng JQ (2008) Microtubules in dendritic spine development. J Neurosci 28:12120-12124.

Hu X, Viesselmann C, Nam S, Merriam E, Dent EW (2008) Activitydependent dynamic microtubule invasion of dendritic spines. J Neurosci 28:13094-13105.

Jaworski J, Kapitein LC, Gouveia SM, Dortland BR, Wulf PS, Grigoriev I, Camera P, Spangler SA, Di Stefano P, Demmers J, Krugers H, Defilippi P, Akhmanova A, Hoogenraad CC (2009) Dynamic microtubules regulate dendritic spine morphology and synaptic plasticity. Neuron 61:85-100.

Ji Y, Lu Y, Yang F, Shen W, Tang TT, Feng L, Duan S, Lu B (2010) Acute and gradual increases in BDNF concentration elicit distinct signaling and functions in neurons. Nat Neurosci 13:302-309.

Kovalchuk Y, Hanse E, Kafitz KW, Konnerth A (2002) Postsynaptic induction of BDNF-mediated long-term potentiation. Science 295:1729-1734.

Marrs GS, Green SH, Dailey ME (2001) Rapid formation and remodeling of postsynaptic densities in developing dendrites. Nat Neurosci 4:1006-1013.

Noritake J, Fukata Y, Iwanaga T, Hosomi N, Tsutsumi R, Matsuda N, Tani H, Iwanari H, Mochizuki Y, Kodama T, Matsuura Y, Bredt DS, Hamakubo T, Fukata M (2009) Mobile DHHC palmitoylating enzyme mediates activity-sensitive synaptic targeting of PSD-95. J Cell Biol 186:147-160.

Rex CS, Lin CY, Kramár EA, Chen LY, Gall CM, Lynch G (2007) Brain- derived neurotrophic factor promotes long-term potentiation-related cytoskeletal changes in adult hippocampus. J Neurosci 27:3017-3029.

Schapitz IU, Behrend B, Pechmann Y, Lappe-Siefke C, Kneussel SJ, Wallace KE, Stempel AV, Buck F, Grant SG, Schweizer M, Schmitz D, Schwarz JR, Holzbaur EL, Kneussel M (2010) Neuroligin 1 is dynamically exchanged at postsynaptic sites. J Neurosci 30:12733-12744.

Sweet ES, Previtera ML, Fernández JR, Charych EI, Tseng CY, Kwon M, Starovoytov V, Zheng JQ, Firestein BL (2011) PSD-95 alters microtubule dynamics via an association with EB3. J Neurosci 31:1038-1047.

Tanaka J, Horiike Y, Matsuzaki M, Miyazaki T, Ellis-Davies GC, Kasai H (2008) Protein synthesis and neurotrophin-dependent structural plasticity of single dendritic spines. Science 319:1683-1687.

Tsuriel S, Geva R, Zamorano P, Dresbach T, Boeckers T, Gundelfinger ED, Garner CC, Ziv NE (2006) Local sharing as a predominant determinant of synaptic matrix molecular dynamics. PLoS Biol 4:e271.

Tyler WJ, Pozzo-Miller LD (2001) BDNF enhances quantal neurotransmitter release and increases the number of docked vesicles at the active zones of hippocampal excitatory synapses. J Neurosci 21:4249-4258.

Viesselmann C, Ballweg J, Lumbard D, Dent EW (2011) Nucleofection and primary culture of embryonic mouse hippocampal and cortical neurons. J Vis Exp pii:2373.

Yoshii A, Constantine-Paton M (2007) BDNF induces transport of PSD-95 to dendrites through PI3K-AKT signaling after NMDA receptor activation. Nat Neurosci 10:702-711. 